\title{
Morphogenesis in Plants: Modeling the Shoot Apical Meristem, and Possible Applications
}

\author{
Eric Mjolsness \\ Jet Propulsion Laboratory \\ California Institute of Technology \\ Pasadena CA 91109-8099 \\ mjolsness@jpl.nasa.gov \\ Victoria Gor \\ Jet Propulsion Laboratory \\ California Institute of Technology \\ Pasadena CA 91109-8099 \\ gor@aig.jpl.nasa.gov
}

\author{
Elliot Meyerowitz \\ Division of Biology \\ California Institute of Technology \\ Pasadena CA 91125 \\ meyerow@its.caltech.edu \\ Tobias Mann \\ Jet Propulsion Laboratory \\ California Institute of Technology \\ Pasadena CA 91109-8099 \\ mann@aig.jpl.nasa.gov
}

\begin{abstract}
A key determinant of overall morphogenesis in flowering plants such as Arabidopsis thaliana is the shoot apical meristem (growing tip of a shoot). Gene regulation networks can be used to model this system. We exhibit a very preliminary two-dimensional model including gene regulation and intercellular signaling, but omitting cell division and dynamical geometry. The model can be trained to have three stable regions of gene expression corresponding to the central zone, peripheral zone, and rib meristem. We also discuss a space-engineering motivation for studying and controlling the morphogenesis of plants using such computational models.
\end{abstract}

\section{Introduction}

In this paper we review the role and structure of the shoot apical meristem in flowering plant morphogenesis, and then suggest an overall approach towards a computational understanding of plant development through gene regulation circuitry. This approach could lead to the ability to thoroughly modify and engineer plant development in biotechnology applications. We exhibit a first, elementary example of such a computational model for one of the essential determinants of morphogenesis in flowering plants, the shoot apical meristem. Finally we speculate on a motivation for studying the biology and bioengineering of plant development in pursuit of self-sustaining space industry, and conclude.

\section{The shoot apical meristem in flowering plants}

\subsection{Role of the meristem}

Shoots and their attendant structures such as leaves and flowers form from a group of stem cells found at the growing tip of the shoot,. called the shoot apical meristem (SAM) [1]. It forms during plant embryogenesis, and after seed germination the SAM remains a collection of undifferentiated cells approximately uniform in shape and size, while providing at its flanks the cells that will become lateral structures (leaves with attendant second-order meristems and flowers), and at its base the cells that will make the stem, including pith and vasculature. Thus, through the life of the plant the addition of cells to the meristem by cell division, and the departure of cells to form differentiated structures, must be closely balanced. Furthermore, the pattern of cell divisions must be highly controlled, to maintain the uniform meristematic shape and to provide flanking structures in appropriate positions (e.g. spiral phyllotaxis).

\subsection{Cellular structure}

The shoot apical meristem, defined as the part of the shoot above the earliest differentiating structures (leaves or flowers), consists of a modest number of cells. In Arabidopsis thaliana, the shoot apical meristem is hemispherical with a radius of $30-40$ micrometers, and 
contains more or less isodiametric cells of average diameter 5 micrometers. Thus there are only 500 to 1.000 cells (Fig. IA). Each cell is surrounded by a primary cell wall of $50 \mathrm{~nm}$ thickness, and the cells divide first by elongation, then septation, whereby a new cell wall is synthesized between two halves of the cell, with the wall dividing the cell in its longest axis. Cells depart from the meristem by differentiation into structures beneath the meristem - thus the meristem moves higher through the life of the plant, and the production of new cells by division must exactly match the departure of old cells via differentiation.

\subsection{Meristematic zones}

Cell division in developing SAMs is complex, but highly patterned. The SAM is divided, generally, into three zones of different cytological appearance: the central zone (or zone of initials), at the apex of the meristem, where cell divisions are infrequent; the peripheral zone, surrounding the central zone, where cell division rates are relatively rapid; and the rib meristem beneath the central zone, where divisions are also rapid [2] (Fig. IC). Leaves and flowers form in the peripheral zone on the flanks of the SAM, and the central cells of the stem originate in the rib meristem. The leaves and flowers form as a result of activation of specific regions in which the planes of new cell walls of subepidermal cells are parallel to the surface (periclinal divisions). The division of cells in the central zone allows for maintenance of the meristem itself, and also provides new cells to the peripheral zone and rib meristem. Continued division of the cells of the rib meristem and the peripheral zone results in the shoot apical meristem moving upwards and leaving older cells behind. This (along with cell elongation) is how the stem grows taller. Thus, we can already see three modes of spatial control of cell division one of slow division in the central zone to maintain the meristem, one of rapid division in the rib meristem and the peripheral zone to make stem, and one that changes the plane of cell divisions in defined locations in the peripheral zone, to make leaf and flower primordia.
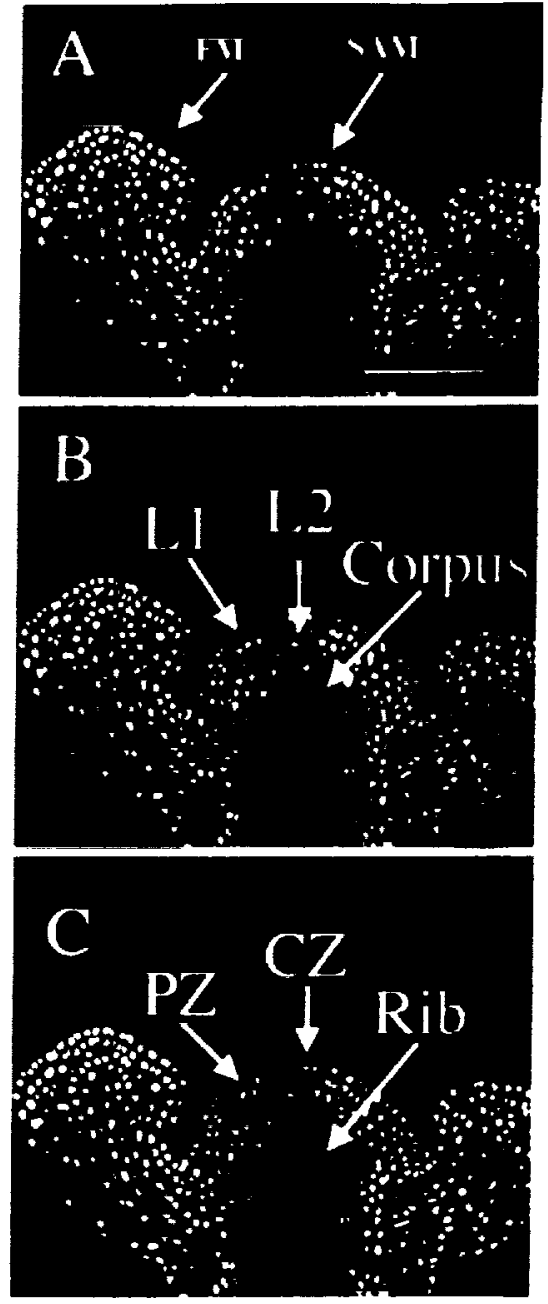

Figure 1. Three vicus of the Arabidopsis thaliana shoot apical meristem. (A.) A laser scanning confocal micrograph showing a central optical section through the shoot apex. The lissue has been stained with propidium iodide, a DNA stain. so nuclei are visualized. FM is a floral meristem arising on the tlank of the SA.M, the shoot apical meristem. (B.) The same photograph as in A., colored in to emphasize the layered structure of the meristem. The Ll (epidermal) and $L ?$ (suhepidermal) layers have exclusively anticlinal planes of eell division. and maintain themselves as clones. The 1.; hou, all planes of eell division. (C.) The pholugraph in 1. collured in to show the raditional runctionsl resum, il the S.A.M. $\mathrm{CZ}$ is central zone, the collection of huwly dividing cells that is the ultimate surce of the entire merstem iand entire aboveground part "It the plant, PZ is peripheral zone, where periclinal cell divinions in the L. l.lvir kide to formation of leaves or thusr. Rih in the rit moristem, where cells that will

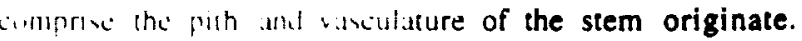
Whut all diwnum in the sime plane lead to files or ribs

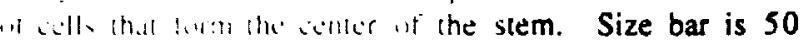
marumbro 


\section{$2.4 \quad$ Meristematic layers}

In addition to the division into zones, the SAM and its descendent structures are divided into clonally distinct layers of cells (Fig. IB) [3][4][5]. There are different numbers of layers in different species; three is the typical dicot number (such as in Arabidopsis). The epidermal cell precursors (or first layer, Ll) form one clone, distinct from the other meristematic cells from the embryo; an almost exclusive pattern of anticlinal cell divisions (with new walls forming perpendicular to the surface) maintains this layer and keeps it distinct from the underlying L2 layer. The L2 is also characterized by anticlinal divisions, and it too remains clorally distinct from other regions. The corpus or L3 contains the remaining cells, which divide in many planes, thereby providing the central cells of stems (including those that will differentiate into the vasculature). All three layers participate in the formation of leaves and of flowers, so that a mature leaf or flower has its epidermis derived from the $\mathrm{Ll}$ layer, subepidermal layers of cells derived from L2, and its central cells (such as at the leaf midrib, or central parts of ovaries) derived from the L3. Thus organ formation as well as meristem maintenance requires the coordinated proliferation of cells in all three layers.

\subsection{The problem}

The overall pattern of SAM cell divisions, starting with the primary shoot apical meristem and resulting in a mature plant, is not at all stereotyped. Genetic mosaics show that there is no fixed pattern of cell lineage except for the general preservation of the clonal layers (e.g. [6][7][8][9]). Indeed, genetic mosaic studies show that there are even occasional violations of the clonal layers, without any consequence for the organization of the plant [5]. Genetic mosaics also show that dividing plant cells communicate division information to each other. In mosaics where cells of the L2 layer are marked by polyploidy (these types of chimeras can be induced by colchicine), a considerable proportion of each leaf blade can be seen to derive from L 2 cells. Similar mosaics where the L 2 is marked by a mutation that prevents chloroplast development (which makes white, nonphotosynthetic leaf cells that divide more slowly than normal) have a much smaller proportion of the leaf derived from the L2, and a much larger contribution of $\mathrm{Ll}$ or of L3 cells than usual [5]. This indicates that the clonally distinct cells communicate division information, and that cells in one clone can alter their division rate and division pattern to accommodate the divisions of their distantly-related neighbors. In addition, the fact that SAMs maintain their size and shape for long periods of time. while their cells continue to divide, indicates that there is some coordination of division between differenl cells.

There is thus much to explain: how does any individual cell know when to divide? Clearly there are cues based on neighboring cells, and perhaps also on distant regions. Superimposed on this is control of the planes of cell division that maintains the clonal layers, and control of the positions where groups of cells divide to form the primordia of leaves, of flowers, and of floral organs.

\section{Modeling the meristem}

The SAM is a complex dynamical system involving at least the following subsystems: regulatory networks of genes and proteins such as transcription factors, receptormodiated signaling, and intracellular signaling pathways; geometrical dynamics owing to elongation, elastic structures in the cell walls and cytosol, and high turgor pressure; and regulated cell division. All this detail is multiplied by the 500 to 1000 cells in the SAM. Thus, it would not be surprising if computational models turn out to be absolutely essential to achieving a scientific understanding of this system. Essential or not, the large number of relevant interacting mechanisms affecting cell division in the SAM means that computational modeling will be helpful. Construction of adequate computational models which can be compared directly to experiment is likely to be of great utility in formulating detailed hypotheses about the dynamics of shoot apical meristems, and in prioritizing competing hypotheses for testing in the laboratory.

Our method for constructing models of morphogenetic systems [10] such as the SAM begins with network models of gene regulation, dynamical grammars to connect up submodels, and machine learning algorithms for fitting the gene network models to data. The resulting "gene circuit" method has met with predictive success in the Drosophila syncytial blastoderm [11][12]. Further elements of the developmental theory were included to model cell-cell signaling in Drosophila [13][14] including automated fits to expression patterns. The original theory also contains provision for dynamical geometry including viscous-elastic dynamics similar to [15], and is easily adapted to include phosphorylation and dephosphorylation networks such as the MAP kinase cascade [16] by replacing some gene circuit differential equations with enzyme kinetics ones. So in principle, each of the major dynamical subsystems affecting meristem gene expression and cell division pattems are amenable to mathematical modeling and computer simulation by the gene circuit method. 


\section{Preliminary Modeling Results}

We have excercised the gene circuit method as previously extended to include intercellular signaling, and constructed a very preliminary model of a two-dimensional slice through the SAM which is in qualitative agreement with gene expression patterns for CLVI and CLV3. This model is similar to and uses the same computer program as the Notch/Delta signaling model for Drosophila neurogenesis of [13][14][17]. Consequently it does not yet include dynamical geometry or cell division, both of which are essential for real meristem functioning and patterning. A major qualitative difference in model output from Drosophila neurogenesis is that in neurogenesis the pronteural fate is adopted by single, isolated cells rather than cells in large contiguous regions as in the meristem. Nevertheless a suitable circuit fit is found in each case.

Figure 2 shows the desired pattern of central zone, peripheral zone, and rib meristem cells on a static hexagonal grid. This pattern was used as the target final condition for training according to the gene circuit method. The initial conditions for training patterns were one of two types: either (a) a highly attentuated version of the final pattern to which noise was added in the form of small random variations in protein levels along with occasional "flips" from one cell type to another, or (b) an uninformative, homogeneous, average starting state with small variations added in.

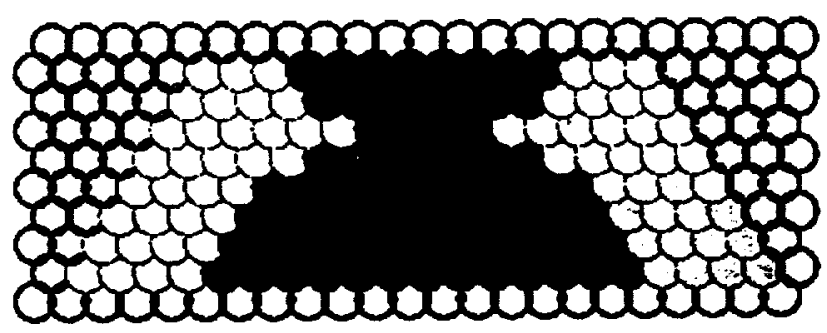

Figure 2. Target final condition, analogous to Figure IC.

Further details of the simulations are as follows. Along the bottom of the meristem pattern, reflecting boundary conditions are imposed to simulate the effect of a much larger grid of cells including shoot below the meristem. The parameters solved for include a $3 \times 3$ connection matrix for the artificial neural net which models all intracellular interactions (including transcriptional regulation) among activated forms of the three gene products favoring the three cell fates; also the six nondiagonal components of a similar matrix representing intercellular interactions (probably receptor-ligand interactions) among the same species.
When trained only with a single type (a) initial condition, the gene network was able to stably converge to a close approximation of the desired linal state, both from the trained initial condition and from other "test" initial conditions taken from the same probability distribution. When trained with one initial condition of type (a) and one of type (b), the gene network was able to converge to final states such as that of Figure 3, in which the type (a) initial condition meristem is correct and the type (b) initial condition meristem is missing its central zone (whose cells are converted to peripheral zone cells) but otherwise has a close approximation to correct peripheral zone and rib meristem. Further computational experiments will be required to determine whether the type (b) meristem can also be correctly trained, perhaps by a slower annealing schedule, or whether additional model features such as cell division are required.

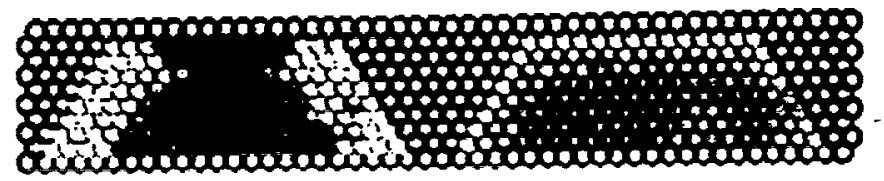

Figure 3. Trained final conditions. (a) Informative initial point. (b) Uninformative initial point.

These computer experiments demonstrate that gene circuits can be automatically inferred by simulated annealing, so as to agree with hemispherical expression patterns suitable for rib meristem (indicated by gene product CLVI) and central zone (CLV3) along with peripheral zone (indicated by a third gene circuit component).

\section{Application: Seed Ships}

For both robotic and human space exploration missions there are strong reasons to learn engineering methods from biological systems. In the longer term, perhaps chief among these reasons is the launch mass required to establish a self-sustaining industrial presence in space. In this section we discuss a long-term and necessarily speculative motivation for studying the biology and bioengineering of plant development in pursuit of self-sustaining space industry.

The non-terrestrial solar system is rich in mineral and energy resouces, many of which reside at small bodies which have relatively shallow gravity wells compared to that of Earth. An optimal way to run a solar system-wide economy might be to keep human habitation concentrated on Earth (where we enjoy the largest terrestrial environment that can be expected for centuries or more), transmit up information such as industrial designs, plans, and encrypted 
command suites into a solar-system wide industrial complex, and receive high-value goods via automated oneway descent transports manufactured in space. In this updated form of mercantilism, the terrestrial economy would also receive massive return information feeds "for science, engineering, and entertainment. Big space projects like the construction of large-scale distributed observatories or, eventually, a launcher for interstellar probes would also become supportable with the solar system energy and materials mobilized by such infrastructure. This scenario minimizes the requirement for launch mass since relatively few people would need to be transported into space, but it still requires some enormous-sounding undertaking to lift whole industries (including construction equipment, assembly lines, etc.) up from the Earth.

The problem is that we are thinking non-biologically. The way plants spread their sophisticated chemical engineering operations throughout a habitat is by using seeds - genetic information, plus a minimal physical and energetic starting point. The rest of the mass and energy for making a new plant comes from local materials wherever the seed germinates. We can't afford to launch a lot of factories or construction machinery from Earth; most equipment needs to be built and/or grown in place using local resources. What would come from Earth instead is small, affordable "seeding" spacecraft and a continual stream of design and control information. This is an enormous advantage which hamessing biological development can bring to space engineering.

As an example, consider agriculture. On Earth the growing tips of plants - the plant "meristems" - are the source for nearly all human food (and are indirectly the source of all of it) and fiber; of all of our cellulose (and thus rayon, paper, etc.) and of substantial quantities of chemical feedstocks, pharmaceuticals, oils, waxes, and even perfumes and cosmetics. This is the kind of technological base we need to establish local, self-sustaining industry in space, at whatever level of human presence turns out to be most useful. And of course plants, oceanic algae and cyanobacteria produce oxygen from carbon dioxide, as is crucial to all animal life on Earth.

If we can control plant development at the level of gene regulation circuitry, as now seems possible by way of computational models extending as those we have presented. then we may be able to modify plants to adapt to altered conditions in greenhouses throughout the solar system. Perhaps more importantly, such plants might be modified to produce new chemical feedstocks and mechanical structures required by local industry with a minimum of investment in launching massive capital equipment. An alternative approach is to use a computational understanding of plant growth and function to create an artificial analog using partly or completely different chemical, mechanical and information technologies. Either way, achieving a scientific and computationally effective understanding of the development of natural plants is likely to be an essential starting point.

\section{Conclusions}

In this paper we have suggested an overall approach towards a computational understanding of plant development through trainable models of gene regulation circuitry and cell-cell signaling. This approach could lead to the ability to thoroughly modify and engineer plant development in biotechnology applications, as required for the space industry scenario we sketched.

\section{References}

[1] Meyerowitz, E.M. (1997) Genetic control of cell division patterns in developing plants. Cell 88, 299-308.

[2] Steeves, T.M and Sussex, I.M. (1989) Patterns in Plant Development, Cambridge.

[3] Satina, S., Blakeslee, AF and AG Avery (1940) Demonstration of the three germ layers in the shoot apex of Datura by means of induced polyploidy in periclinal chimeras. Amer. J. Bot. 27, 895-905.

[4] Satina, S. and Blakeslee, A.F. (194l) Periclinal chimeras in Datura stramonium in relation to development

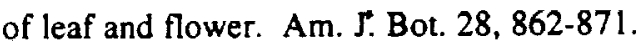

[5] Tilney-Bassett, R. A. E. (1986). Plant Chimeras, E. Arnold, London.

[6] Irish, V. F. and Sussex, I. M. (1992). A fate map of the Arabidopsis embryonic shoot apical meristem. Development 115, 745-753.

[7] Furner, I. J. and Pumfrey, J. E. (1992). Cell fate in the shoot apical meristem of Arabidopsis thaliana. Development 115, 755-764.

[8] Furner, I. J. and Pumfrey, J. E. (1993). Cell fate in the inflorescence meristem and floral buttress of Arabidopsis thaliana. Plant J.4, 917-931.

[9] Bossinger, G. and Smyth, D.R. (1996) Initiation patterns of flower and floral organ development in Arabidopsis thaliana Development, 122, 1093-1102. 
[10] Mjolsness, E., Sharp. D.H., and Reinitz, J., (1991) A Connectionist Model of Development, Joumal of Theorefical Biology, 152, 429-454.

[11] Reinitz, J., Mjolsness, E., and Sharp, D.H. (1992) Model for Cooperative Control of Positional Information in Drosophila by bed and Matemal $h b$, Joumal of Experimental Zoology 271, 47-56 1995. Also Technical Report LAUR-92-2942 Los Alamos National Laboratory 1992.

[12] J. Reinitz and D. H. Sharp, "Mechanism of eve Stripe Formation". Mechanisms of Development 49:133-158, 1995.

[13],Marnellos, G., and E. Mjolsness, "A Gene Network Approach to Modeling Early Neurogenesis in Drosophila", in Pacific Symposium on Biocomputing, eds Altman, Dunker, Hunter, and Klein, pp. 30-41,World Scientific, 1998

[14] Marnellos, G. and E. Mjolsness, "Probing the Dynamics of Cell Differentiation in a Model of Drosophila Neurogenesis". Artificial Life VI: Proceedings of the Sixth International Conference on Artificial Life, eds. C. Adami, R. Belew, H. Kitano, and C. Taylor, pp. 161-170, MTT Press 1998.

[15] Odell, G., Oster, G.F., Burnside, B., Alberch, P. (1981) Devel. Biol. 85:446-462

[16] C-Y F. Huang and J. E. Ferrell, Jr. "Ultrasensitiviity in the Mitogen-Activated Protein Kinase Cascade", Proc. Natl. Acad. Sci. USA 93:10078-10083, September 1996.

[17] Marnellos, G.,"Gene Network Models Applied to Questions in Development and Evolution". Ph.D. Disseration, Yale University, 1997. 